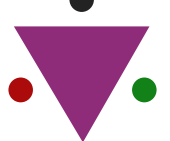

IJCRR

Section: Healthcare

Sci. Journal Impact

Factor: $6.1(2018)$

ICV: 90.90 (2018)

(c) (1) (3)

Copyright@IJCRR

\title{
Rehydration of Air-dried Smears for Papanicolaou Staining in Cytopathological Section of Department of Pathology
}

\section{Shweta Bahadure ${ }^{1}$, Pragati M. Shahare², Sunita Vagha ${ }^{3}$}

\begin{abstract}
'Assistant Professor, Department of Pathology, Jawaharlal Nehru Medical College, Datta Meghe Institute of Medical Sciences, Sawangi (M) Wardha, Maharashtra, India; ${ }^{2}$ MSc. MLT 2nd year, Department of Pathology, Jawaharlal Nehru Medical College, Datta Meghe Institute of Medical Sciences, Sawangi (M) Wardha, Maharashtra, India; ${ }^{3}$ Professor and HOD, Department of Jawaharlal Nehru Medical College, Datta Meghe Institute of Medical Sciences, Sawangi (M) Wardha, Maharashtra, India.
\end{abstract}

\section{ABSTRACT}

Background: Wet fixed smears are commonly used for routine investigation in determining many precancerous and cancerous lesions. Wet fixed smears are associated with few side effects which leads to diagnostic difficulties. Therefore the present study is used to determine the effects of rehydration on air-dried smears by comparing cellular preservation and staining quality in rehydration smears and wet fixed smears.

Objectives: 1) To compare the cytomorphological features in conventional Pap smears and rehydration air-dried Pap smear. 2) To compare the staining quality in rehydration smears and wet fixed smears. 3) To identify if rehydration techniques can be used as an alternative method particularly in rural setup or in resource-limited settings.

Methods: Our study is a prospective analytical study to be conducted in Cytopathology section of the Department of Pathology. 100 fluid sample were examined during the study period. Wet fixed smears are processed routinely. Air-dried smears are rehydrated with normal saline for thirty seconds and immediately added to the jar containing fixative $95 \%$ ethanol for thirty minutes. Both the smears were then processed and stained by the Papanicolaou technique. Both the smears will be screened, assessed and graded for various cytomorphological parameters. The cervical smears will be reported as per the 2014 Bethesda system.

Expected Results: Statistically significant similarities can be observed in between two groups one with wet fixed smears and other with rehydrated air-dried smears when compared for various cellular features. On comparing two groups for its cytological diagnosis, results can be found out in the agreement. The rehydrated air-dried technique can be used effectively for FNACs, Effusion fluids, Exfoliated cells. Interpretation of rehydrated air-dried smears and staining quality is far better when compared to conventional wet smears.

Conclusion: The conclusion will be based on the results of the study.

Key Words: Cervical cytology, Pap smears, Rehydration smears, Wet fixed smears

\section{INTRODUCTION}

In cytopathology practice, two types of smears are used for making the diagnosis. ${ }^{1}$ One is wet smears, which is alcohol fixed and is stained by pap stain or hematoxylin \& eosin stain and the other one is air-dried smear which is stained by Romanowsky stain. ${ }^{2}$ Wet fixed smears (pap smears) and air-dried smears are used for early detection of cancer lesions and any inflammatory lesions in Cytopathology. Pap smears are stained with Papanicolaou stain. It is considered as the best stain for determining nuclear features including chro- matin pattern. Screening with the help of pap examination of cervical smears is used for early detection of premalignant and malignant lesion of the uterine cervix. Wet smears are also used for diagnosis of various lesions on FNAC (fine needle aspiration cytology) and cytological examination of body fluids. ${ }^{2}$

Pap smears are commonly used for diagnosis of cervical lesions. In routine practice, the sample is collected from cervix with the help of Ayer's spatula and transferred to the slide by smearing spatula on the slide. The smears are then fixed

\section{Corresponding Author:}

Miss. Pragati M. Shahare, MSc. MLT 2nd year, Department of Pathology, Jawaharlal Nehru Medical College, Sawangi Meghe, Wardha, Maharashtra, India; Mobile: 8411043799; E-mail: pragatishahare20@gmail.com

ISSN: 2231-2196 (Print)

Received: 20.09 .2020
ISSN: $0975-5241$ (Online)

Revised: 24.10 .2020
Accepted: 13.11 .2020
Published: 30.11 .2020 
immediately in $95 \%$ ethanol in a Kopplin jar and processed in the laboratory. The smears are stained with Papanicolaou stain and then examined by a cytotechnologist and further by cytopathologist for identification of any inflammatory, infectious, premalignant and malignant lesions. Smears are collected by gynaecologists and the trained staff in hospital setup whereas many times it is collected by untrained paramedical staff in primary health centres or during screening programmes. Improper fixation of wet smears and drying artefacts are common if the smears are collected by untrained staff or in case of heavy workload. Air drying artefact is commonly seen in setups where the staff who collects the smears don't have adequate training in executing the task.

Wet fixed smears are also associated with few disadvantages like floating of cells from the slide which is prevented by albuminisation of slides. Albuminisation causes heavy green background which makes the interpretation difficult. All over it leads to diagnostic difficulties. It requires the collection of repeat smears, which increases workload over clinical and laboratory setup and also causes discomfort to patients. ${ }^{3,5}$ Wet fixed smears followed by pap staining are routinely used for better visualization of nuclear morphology, whereas air-dried smears followed by Romanowsky staining is used for observing cytoplasmic details. Rehydration of air-dried smears has been suggested by many workers as an alternative technique to conventional pap smears in cytology to overthrown the problems of poor fixation and air drying artefacts. ${ }^{6-9}$ Studies over rehydration of air-dried smears are scarce in the literature. Therefore the present study is undertaken to determine the effect of rehydration on air-dried smears by comparing cytoplasmic and nuclear details and staining quality of rehydrated smears and conventional pap smears.

\section{OBJECTIVES}

1) To compare the cytomorphological features in wet fixed smears and rehydrated air-dried smear.

2) To compare the staining quality in rehydration smear and wet fixed smears.

3) To identify if rehydration techniques can be used as an alternative method particularly in rural setup or in resource-limited settings.

\section{MATERIALS AND METHODS}

Our study is an analytical prospective study to be conducted in Cytopathology, the section of the Department of Pathology, Jawaharlal Nehru Medical College, Sawangi (Meghe), Wardha. Approval was obtained from the institutional Ethics committee and informed consent was taken from the patients participating in this study.
Study design - Observational, prospective and analytical

Study period - November 2019 to November 2020

Sample size - 100 (calculated by Krejcie and Morgan formula)

\section{Inclusion criteria:}

1. All the FNAC smears which are air-dried

2. Exfoliative cytological samples including cervical smears, BAL fluid, urine cytology.

3. Effusion fluids including ascitic fluid. pleural fluids, pericardial fluids.

\section{Exclusion criteria:}

1. Pregnant women

2. Menstruating women

3. Women on chemotherapy

Throughout one year hundred, smears were collected from females who visited in the gynaecological and non-gynaecological section of Acharya Vinoba Bhave rural hospital, a tertiary care centre. Patients came to visit the hospital either for a routine checkup or with any clinical indication has been included in the study. The age of the patients included in the study was from 15 to 80 years. Prior informed consent will be taken from the patients participating in the study. FNAC air-dried smear, body fluid smears, cervical smears will be collected in cytopathology section of Department of Pathology. Two sets of smears were made, out of which one is fixed in $95 \%$ ethanol and the other set was allowed to air dry for thirty minutes to two hours. Air-dried smears are rehydrated with normal saline for 30 seconds and immediately added to the jar containing fixative $95 \%$ ethanol for thirty minutes. The smears from both sets were then stained by the Papanicolaou technique. Then the slide will be examined by the cytopathologist. Both the smears will be screened, assessed, and graded for various cytomorphological parameters. the cervical smears will be reported as per the 2014 Bethesda system. The staining quality of smears was assessed by applying given parameters and graded accordingly.

a) Determining cellularity - high/intermediate/low

b) Identifying cytolysis in the smear and graded it accordingly $-0+/ 1+/ 2+/ 3+$

c) Checking for cell borders - distinct/indistinct

d) Looking into staining quality of cytoplasm- excellent/ satisfactory/ unsatisfactory

e) Noting nuclear borders- distinct/indistinct

f) Looking for nuclear chromatin- hazy/crisp

g) Looking for the presence of red blood cells and neutrophils particularly in cervical smears and graded accordingly $-0+/ 1+/ 2+/ 3+$ 
The criteria given above are applied to all smears including, FNAC smears, exfoliative smears, effusion cytological smears. Within cervical smears, both squamous cells and glandular cells are graded according to above-given criteria. Conventional Wet fixed smears and rehydrated smears were categorized as satisfactory and unsatisfactory and comparison between the two was made accordingly. Statistical analyses will be done by using Chi-square $\left(\mathrm{x}^{2}\right) /$ fisher exact test, to determine the significance of the difference between the two groups. Results will be considered significant if the value was $<0.05$.

\section{EXPECTED RESULTS AND OUTCOMES}

Statistically, significant similarities can be observed in between two groups one with wet fixed smears and other with rehydrated air-dried smears when compared for its cellularity, amount of cytolysis, nuclear borders and chromatin, quality of cytoplasmic staining, background clearing and cell borders. On comparing two groups for its cytological diagnosis, results can be found out in the agreement. All kind of inflammatory lesions, infectious conditions, various premalignant and malignant lesions can be easily diagnosed with rehydrated air-dried smears. As the duration of air drying increases, the quality of staining becomes less satisfactory for evaluation. The rehydrated air-dried technique can be used effectively for FNACs, Effusion fluids, Exfoliated cells. Interpretation of rehydrated air-dried smears and staining quality is far better when compared to conventional wet smears.

\section{DISCUSSION}

In developing countries particularly in a rural setup, mortality and morbidity due to cancer are very high. Screening is an important step to prevent various premalignant and malignant lesions particularly related to female genital tract ( examination of cervical smears in sexually active women). Knowledge of collecting and processing, cervical smears is of utmost importance for the diagnosis of premalignant lesions of the cervix. Routinely wet smears are fixed immediately in $95 \%$ ethanol. But is commonly associated with drying artefacts, because the collection is carried out by workers who don't have essential training in fulfilling the task. If there is a delay in the fixation it will lead to many pitfalls like the disintegration of cells, interference in various chemical processes such as staining, bluing and clearing, putrefaction of cells. This leads to diagnostic difficulties. Moreover, these patients required repeat smears which added workload for clinical and personnel laboratory., ${ }^{3,5}$ Various studies have been carried out to determine the importance of rehydration air-dried technique, whether it could replace conventional wet fixation technique. Many rehydrating agents are used by different authors like normal saline, tap water, glycerin. . $^{1,2,10}$ It was first discovered by Lencioni et al in 1954, where he used tap water as a rehydrating agent. ${ }^{6}$ Subsequently many studies show favourable results on rehydration of air-dried smears. ${ }^{7,8,10}$ Different studies concluded normal saline as a best rehydrating agent. ${ }^{2,3,10}$ Many studies have been suggested that rehydration up to twenty-four hours shows satisfactory results. ${ }^{6-8}$ Whereas few studies suggested that rehydration done within two hours give the best results. ${ }^{7}$ Immersion of smears in rehydrating agent up to thirty seconds give appropriate results as longer immersion of smears in rehydrating agents can lead to certain morphological changes like wrinkling of the nucleus. ${ }^{11}$ Sivaraman et al suggested that air-dried smears can be kept for up to four days before rehydration without compromising cellular details. ${ }^{7}$

Sivaraman et al found that rehydrating smears decreased the rate of unsatisfactory smears by $2 \%$. According to him rehydration is not superior to conventional wet fixed smears, but are equally good. He concluded that rehydration before two hours has superior effects when compared to rehydrating smears after two hours. ${ }^{7}$ Chan et al proved that optimal time for rehydrating smears is 30 minutes but Sivaraman found that air drying up to two hours can also provide better results. ${ }^{3}$ Dahlstrom et al found that results on exfoliated gastric cells are satisfactory even after air-drying for four hours. ${ }^{5}$

Presence of red blood cells in the background can mask the interpretation of smears. Rehydrating smears lead to lysis of red blood cell making the background clear and interpretation easier similar to previous findings. ${ }^{3,4,7}$ Cytoplasmic staining is found to be better with rehydrated smears as compared to conventional wet smears. ${ }^{2,5,7,11}$ Many of the authors noted no significant differences in nuclear staining with rehydrated smears and conventional wet fixed smears. Both of the technique shows satisfactory results in regards to nuclear features. Few authors found that rehydrated air-dried technique was superior in determining anisonucleosis within tumour cells and in detecting nucleoli within malignant cells in both squamous and glandular cells..$^{6,9,11}$ Generally size of cell and nucleus with conventional pap smear are smaller when compared to Romanowsky stain therefore detection of anisonucleosis and anisokaryosis in malignant cells are inferior with conventional pap smears as compared to Giemsa stain. This effect is compensated by rehydration technique as here air dried smears are used.

Cellularity is high with rehydration air dried Papanicolaou smears as compared to conventional papanicolaou smears. ${ }^{1,2}$ Overall quality is better with rehydrating Papanicolaou smears when compared with conventional Papanicolaou smears. ${ }^{1,27,10,11}$ Factors which may lead to improve quality with rehydrating smears are preventing air drying artifact, floating of cells in thick specimen when they are fixed immediately can be avoided with rehydrating smears, lysis of red 
blood cells on rehydration. ${ }^{7,11}$ Refrigeration in cases when the processing of rehydration was delayed also shows equivalent results as that of rehydration of air-dried smears within two hours. ${ }^{7}$ Smears can be refrigerated up to twenty-four hours before processing, shows satisfactory staining quality. Refrigeration of air-dried smears can be used routinely in the practice, particularly in the setup where immediate processing of smears is not possible.?

There are many advantages of rehydrating smear: technique is simple, rapid results can be obtained, inexpensive, floating of cells can be prevented which is a side effect of immediate fixation, cells have better adherence to the slides, cells appear to be flatter in rehydrated smears, depth of focus of nuclei is shallower which helps in taking microphotographs. We can use this technique for other staining methods like IHC technique, hematoxylin and eosin staining method, and also Giemsa staining. ${ }^{7,11}$

\section{Limitations:}

- Interobserver variability.

- Technical errors while processing can influence the results.

\section{CONCLUSION}

Rehydration air-dried technique can be used as a satisfactory alternative for conventional wet fixation method, which can be followed routinely or in conjunction with conventional wet fixed smears.

\section{ACKNOWLEDGMENT}

Authors acknowledge the immense help received from the scholars whose articles are cited and included in references to this manuscript. The authors are also grateful to authors / editors / publishers of all those articles, journals, and books from which the literature for this article has been reviewed and discussed.

\section{Conflict of Interest: Nil}

Source of Funding: Nil

\section{REFERENCES}

1. Gupta S, Sodhani P, Chachra KL. Rehydration of air-dried cervical smears: a feasible alternative to conventional wet fixation. Obstet Gynecol 2003 Oct;102(4):761-4.

2. Kapse SS, Arakeri SU, Yerranguntla DP. Rehydration of airdried smears with normal saline: An alternative for conventional wet fixation method in the cervical cytological study. J Cytol 2018 Oct;35(4):199.

3. Chan JK, Kung IT. Rehydration of air-dried smears with normal saline: application in fine-needle aspiration cytologic examination. Am J Clin Pathol 1988 Jan 1;89(1):30-4.

4. Ng WF, Choi FB, Cheung LL, Wu C, Leung CF, Ng CS. Rehydration of air-dried smears with normal saline. Application in fluid cytology. Acta Cytologica. 1994 Jan;38(1):56-64.

5. Dahlstrom JE, Holdsworth J, Bassett ML, Jain S. Rehydration of air-dried smears. An alternative method for cytologic analysis of exfoliative cells. Acta Cytologica 1999 Mar;43(2):214-7.

6. Lencioni LJ, Staffieri JJ, Cardonnet LJ. Vaginal and urinary msediment smear staining technique without previous fixation: Adapted to Papanicolaou's and Shorr's Staining Methods. J Lab Clin Med 1954;44(4):595-9.

7. Sivaraman G, Iyengar KR. Rehydrated air-dried Pap smears as an alternative to wet-fixed smears. Acta Cytologica 2002;46(4):713-7.

8. Bales CE, Durfee GR. Cytologic techniques: Part-I. In: Koss LG, ed. Diagnostic cytology and its histologic basis. $4^{\text {th }}$ ed. Philadelphia: Lippincott, 1992:1451-3.

9. Atkinson KM. Specimen collection and determinants of adequacy. Gynaecologic Cytopathology. Philadelphia, LippincottRaven. 1997:7-18.

10. Zafer A. Rehydration with corn syrup of air dried buccal smear: An alternative to routine wet fixation. Periodon Prosthodon 2017,3:1.

11. Zare-Mirzaie A, Abolhasani KK. Rehydration of air-dried cervical smears: an alternative to routine wet fixation. Acta Medica Iranica 2007:365-8. 